\title{
Impact of Telehealth Visit and Socioeconomic Status (SES) on Hydroxyurea Response in Sickle Cell Anemia
}

\author{
Sarah Shaner ${ }^{1}$, Lee Hilliard ${ }^{2}$, Thomas Howard ${ }^{3}$, Brandi Pernell ${ }^{3}$, Smita Bhatia ${ }^{2}$, and \\ Jeffrey Lebensburger ${ }^{3}$ \\ ${ }^{1}$ The University of Alabama at Birmingham School of Medicine \\ ${ }^{2}$ University of Alabama at Birmingham \\ ${ }^{3}$ University of Alabama-Birmingham
}

May 7, 2021

\begin{abstract}
Background It is important to ensure access to hydroxyurea (HU) for patients with sickle cell anemia (SCA) living in rural areas without easy access to experts in sickle cell management. The UAB Pediatric Sickle Cell program's satellite clinics reduce the barrier of transportation to the university-based clinic. However, as compared to the university clinic, these satellite clinics do not offer immediate access to HU dosing laboratory results. Therefore, a nurse clinician calls families with HU dose adjustments after the completion of the clinic visit. This study evaluated the impact of telehealth dosing adjustments on HU laboratory and clinical response as compared to university-based patients. Methods A one year retrospective chart reviewed was performed to evaluate HU laboratory and clinical response based on clinic location and socioeconomic status for patients with SCA. We identified the number of clinic and acute care visits for one year and calculated the mean CBC and HbF values for each patient. Results We identified 107 academic center participants with SCA prescribed HU and 65 satellite clinic participants. We identified no difference in $\mathrm{HbF}, \mathrm{Hb}, \mathrm{MCV}$, or $\mathrm{ANC}$ by clinic location. We also identified no difference in hospital admissions based on clinic location. Finally, mean socioeconomic indicators by zip code were lower in satellite clinic patients but not associated with a difference in $\mathrm{HbF}$ response. Conclusions The use of telehealth did not negatively impact laboratory response to HU. Future studies should identify novel approaches to improve access to HU among patients with SCA living in rural areas.
\end{abstract}

\section{Impact of Telehealth Visit and Socioeconomic Status (SES) on Hydroxyurea Response in Sickle Cell Anemia}

Shaner, Sarah ${ }^{1}$, Hilliard, Lee² ${ }^{2}$ Howard ,Thomas², Pernell, Brandi², Bhatia ,Smita², and Lebensburger Jeffrey ${ }^{2}$.

${ }^{1}$ University of Alabama at Birmingham School of Medicine, Birmingham, AL

${ }^{2}$ Division of Pediatric Hematology and Oncology, University of Alabama at Birmingham, Birmingham, AL

\section{Correspondence:}

Jeffrey Lebensburger, DO, MSPH

$16007^{\text {th }}$ Ave. S., Lowder Building, Suite 512, Birmingham AL 35233

Phone: (205) 638-9285

Fax: (205) 975-6377

jlebensburger@peds.uab.edu 


\section{Word Count}

Abstract: 250

Main Text: 2223

Tables: 4

Figures: 0

\section{Supporting Information: 0}

Running Title: Impact of Telehealth on HU Response

Key Words : Sickle Cell Disease, Hydroxyurea, Telehealth, Socioeconomic Status

\begin{tabular}{ll}
\hline Abbreviation & Full Term \\
\hline SCA & Sickle cell anemia \\
HU & Hydroxyurea \\
UAB & University of Alabama at Birmingham \\
HbF & Fetal hemoglobin \\
SES & Socioeconomic status \\
CBC & Complete blood count \\
EMR & Electronic medical record \\
ANC & Absolute neutrophil count \\
ED & Emergency department \\
Hb & Hemoglobin \\
MCV & Mean corpuscular volume \\
\hline
\end{tabular}

Portions of this manuscript were presented as an abstract titled "Outcomes of Hydroxyurea Dose Adjustment Via Telehealth" at the $62^{\text {nd }}$ ASH Annual Meeting and Exposition, December 5-8 2020.

\section{Abstract}

\section{Background}

It is important to ensure access to hydroxyurea (HU) for patients with sickle cell anemia (SCA) living in rural areas without easy access to experts in sickle cell management. The UAB Pediatric Sickle Cell program's satellite clinics reduce the barrier of transportation to the university-based clinic. However, as compared to the university clinic, these satellite clinics do not offer immediate access to HU dosing laboratory results. Therefore, a nurse clinician calls families with HU dose adjustments after the completion of the clinic visit. This study evaluated the impact of telehealth dosing adjustments on HU laboratory and clinical response as compared to university-based patients.

\section{Methods}

A one year retrospective chart reviewed was performed to evaluate HU laboratory and clinical response based on clinic location and socioeconomic status for patients with SCA. We identified the number of clinic and acute care visits for one year and calculated the mean $\mathrm{CBC}$ and $\mathrm{HbF}$ values for each patient.

\section{Results}

We identified 107 academic center participants with SCA prescribed HU and 65 satellite clinic participants. We identified no difference in $\mathrm{HbF}, \mathrm{Hb}, \mathrm{MCV}$, or $\mathrm{ANC}$ by clinic location. We also identified no difference in hospital admissions based on clinic location. Finally, mean socioeconomic indicators by zip code were lower in satellite clinic patients but not associated with a difference in $\mathrm{HbF}$ response.

\section{Conclusions}


The use of telehealth did not negatively impact laboratory response to HU. Future studies should identify novel approaches to improve access to HU among patients with SCA living in rural areas.

\section{Introduction}

Health disparities have been shown to exist in access to hematology care for patients with sickle cell anemia (SCA) living in Alabama, especially in rural areas of the state. ${ }^{1}$ To overcome the barrier of transportation to the academic center in Birmingham, AL, the University of Alabama at Birmingham (UAB) Pediatric Sickle Cell Program developed satellite clinics throughout the state. ${ }^{2}$ With the exception of transfusion therapy, patients living with sickle cell can receive care at satellite clinics in Montgomery, Opelika, and Tuscaloosa, AL. One clinical difference in available services at satellite clinics, as compared to the UAB clinic, is that patients receiving hydroxyurea (HU) therapy have their labs obtained on the day of their clinic visit, but CBCs are performed in a local lab and faxed back to the UAB Hematology office and fetal hemoglobin (HbF) levels are processed in Birmingham when the medical team returns to the UAB campus. Therefore, sickle cell providers do not have immediate access to HU dosing labs during the satellite clinic visit. To address this barrier of conducting $\mathrm{HU}$ monitoring clinic visits without immediate access to labs, we developed a telehealth model in which a nurse receives the faxed laboratory results, evaluates the laboratory values relevant to our institutional HU dosing guidelines, confirms dosing changes to achieve maximal tolerated dose with a sickle cell provider, and then calls the patients to review laboratory results and educates on any changes to the HU dosing plan.

Recent data identified that titrating HU to a maximal tolerated dose leads to superior clinical outcomes as compared to lower dose regimens. ${ }^{3}$ Therefore, sickle cell centers should ensure that patients with SCA achieve their maximally tolerated dose of HU. Novel techniques are being developed so that patients can be initiated on a $\mathrm{HU}$ dose that is at or near their maximal tolerated dose. ${ }^{4,5}$ Without this novel approach to initiate $\mathrm{HU}$ at a maximal tolerated dose, clinical centers often achieve maximal tolerated dosing and a high $\mathrm{HbF}$ level by titrating $\mathrm{HU}$ doses at each visit based on CBC results, often targeting a specific neutrophil count. $^{6,7}$ Unique barriers may exist for programs prescribing HU in satellite clinics and/or using telehealth or telemedicine for dose adjustments if labs are not readily available.

Higher $\mathrm{HbF}$ levels and $\mathrm{HU}$ dosing have been achieved in other institutions and during clinical trials as compared the mean levels at UAB; therefore, our program identified a systematic need to improve HU response to therapy among our patient population. ${ }^{6,8}$ Prior to implementing any novel approaches to improve adherence, we performed this study to determine if systematic barriers exist in HU dosing adjustments based on our telehealth plan for satellite clinics. We hypothesized that telemedicine dosing, where the medical provider does not review laboratory results and make in-person $\mathrm{HU}$ dose adjustment at the time of their medical visit, would not result in lower HU response to therapy. To test this hypothesis, we evaluated the HU dosing and adherence laboratory values for patients cared for at both the UAB Pediatric Sickle Cell Clinic at Childrens of Alabama and satellite clinics over a one year period. We also evaluated the association between zipcode based socioeconomic indicators on HU response outcomes.

\section{Methods}

We performed a one-year retrospective review of $172 \mathrm{HbSS}$ and $\mathrm{HbSb0}$ thalassemia patients prescribed $\mathrm{HU}$ during 2019 that were cared for in either the academic center (Birmingham) or satellite clinics (Montgomery, Tuscaloosa, Opelika). Patients prescribed HU older than age 18 at the beginning of 2019 were excluded. We excluded two patients with HbSD, twenty-two patients with $\mathrm{HbSC}$, and seven patients with $\mathrm{Hb} \mathrm{Sb}+$ thal. We excluded SCA patients on HU and a second sickle cell disease modifying therapy, such as chronic transfusion therapy or an investigational or novel SCA drug therapy. We abstracted from the EMR: age, sex, clinic location, diagnosis, HU dose $(\mathrm{mg} / \mathrm{kg})$, white blood cell count, hemoglobin, mean corpuscle volume, platelet count, absolute reticulocyte count, hemoglobin F, and absolute neutrophil count from each clinic visit. We recorded the total number of well-clinic visits and categorized adherence to clinic visits/year as [?] 2 or $>2$; satellite clinic visits are fixed either monthly to every three months which limited our ability to reschedule patients with missed visits. Well-clinic visits were limited to HU follow-up appointments only. 
We recorded the number of ED visits and UAB pediatric pain clinic visits and noted if the chief complaint included sickle cell related pain. We also recorded the number of admissions to the hospital for a) pain and b) all other sickle cell complications except for scheduled procedures. We collected acute visit data from the EMR at the academic center for Birmingham clinic participants and from patient recall from satellite clinic participants. We evaluated dosing adjustment as whether dosing was increased for ANC $>4000$ per institutional standard of care. We categorized correct dosing adjustments if $>65 \%$ of visits had a dose adjustment per institutional protocol; we categorized patients as a deviation from protocol if the physician did not increase the dose based on ANC. We defined non-adherence to $\mathrm{HU}$ as a $\mathrm{HbF} \%<5$. Socioeconomic variables were assessed using the home address provided by the participant and analyzing the information available for that geographic location. Home address information was linked with the census track data to determine the poverty level and the mean household income for the census track of the patient's residence.

Our primary outcome for this study was percent fetal hemoglobin: our primary predictors were clinic location, age, and socioeconomic status. Our secondary outcomes were difference in hydroxyurea monitoring labs, clinic visits, and acute care utilization. We performed descriptive statistics including mean and standard deviation (s.d.), t-test for continuous variables, chi-square for categorical variables, and regression modelling using JMP Pro 14 (Cary, NC). P-values $<0.05$ were considered statistically significant.

\section{Results}

\section{Study population}

We identified 107 participants prescribed $\mathrm{HU}$ at the academic center and 65 participants from satellite clinics. The mean age ( \pm s.d.) of the 172 participants was $11 \pm 5$ years. Fifty two percent of participants were female; we did not identify a mean difference in sex of participants by clinic location $(\mathrm{p}=0.9)$. The mean ( \pm s.d.) $\mathrm{Hb}$ was $8.5 \pm 1.1 \mathrm{~g} / \mathrm{dL}, \mathrm{MCV}$ was $91.3 \pm 10.1 \mathrm{fL}, \mathrm{HbF} \%$ was $12.7 \pm 6.7$, and $\mathrm{ANC}$ was $4.9 \pm 2.3 \times 10^{3} / \mathrm{L}$. The mean poverty level of all the communities our participants live in was $18.1 \pm 4.2$, the mean ( \pm s.d.) median household income was $50,119 \pm 8,298$, the mean percent of adults with less than a high school diploma was $13.5 \pm 3.9 \%$, and the mean percent of adults with a high school diploma only was $29.1 \pm 5.0 \%$ (Table 1 ).

\section{Laboratory and clinical outcomes by clinic location}

We identified no significant differences in HU monitoring laboratory values for $\mathrm{Hb}(\mathrm{p}=0.6), \mathrm{MCV}(\mathrm{p}=0.7)$, $\operatorname{HbF}(\mathrm{p}=0.13)$, ANC $(\mathrm{p}=0.9)$ by clinic location (Table 2$)$. We did not identify a difference in all admissions to the hospital $(\mathrm{p}=0.5)$ or pain admissions to the hospital $(\mathrm{p}=0.6)$ between academic center vs. satellite clinic patients. However, academic center patients did have a higher number of acute pain visits $(\mathrm{p}=0.03)$ to either the ED or UAB pediatric pain clinic (Table 3). As expected, among all participants, non-adherence to $\mathrm{HU}(\mathrm{HbF}<5 \%)$ was associated with the highest number of admissions ( $\mathrm{p}=0.02)$. Among 149 patients with more than one clinic visit, $97(65 \%)$ patients had more than $65 \%$ of clinic visits with a correct dose adjustment; we identified no statistical difference in correct dosing adjustment among Birmingham patients $(67 \%)$ and satellite clinic patients $(62 \%, \mathrm{p}=0.5)$. Patients cared for at the academic center had a statistically higher number of clinic visits $(\mathrm{p}=0.01)$ and more patients that attended $>2$ clinic visits. $\mathrm{HbF}$ response was significantly associated with the number of clinic visits $(\mathrm{p}=0.03)$. Finally, HbF response was significantly inversely correlated with age $(\mathrm{p}=0.003)$. Adjusting for clinic location, number of clinic visits, and age, $\mathrm{HbF}$ was associated with age $(\mathrm{p}=0.006)$ and clinic visits $(\mathrm{p}=0.006)$, but not associated with clinic location.

\section{Difference by Socioeconomic Category}

We identified significantly worse socioeconomic indicators for patients who attend our satellite clinics as compared to our UAB clinic for poverty level (20.3 \pm 4.2 vs $16.8 \pm 3.6, \mathrm{p}=0.0001)$, median household income $(\$ 45,704 \pm 7,048$ vs $\$ 52,841 \pm 7,853 \mathrm{p}=0.0001)$ and the percent of adults in the community with less than a high school diploma ( $15.6 \pm 3.5$ vs $12.2 \pm 3.6, \mathrm{p}=0.0001$ ) (Table 4$)$. When analyzing HbF by socioeconomic data, we did not identify an association between $\mathrm{HbF}$ levels with poverty level $(\mathrm{p}=1.0)$, median household income $(\mathrm{p}=0.8)$ or percent of adults in the community with less than a high school diploma $(\mathrm{p}=0.7)$. Finally, we repeated our regression analysis to determine if community poverty level or median household income 
impacted $\mathrm{HbF}$ value in addition to our prior variables of clinic location, age, and number of clinic visits. Again, only number of clinic visits $(\mathrm{p}=0.02)$ and age $(\mathrm{p}=0.003)$ significantly impacted $\mathrm{HbF}$ but clinic location did not significantly impact $\mathrm{HbF}$ when including socioeconomic data.

\section{Discussion}

It is important for patients with sickle cell anemia prescribed $\mathrm{HU}$ to achieve a high $\mathrm{HbF}$ and maximal tolerated dose. A recent randomized controlled trial demonstrated that titration to maximal tolerated dose of $29.5 \mathrm{mg} / \mathrm{kg} /$ day is clinically superior to fixed dose $\mathrm{HU}$ at $19.2 \mathrm{mg} / \mathrm{kg} /$ day dosing and associated with an almost $50 \%$ higher $\mathrm{HbF}$ level. ${ }^{3}$ While our clinical program identified lower levels of HU response than randomized clinical trial data, our data did not demonstrate a systematic barrier to achieving a high $\mathrm{HU}$ dose and high $\mathrm{HbF}$ response based on the use of telehealth dosing or for aggregate socioeconomic data. However, we identified one potential systematic barrier for our satellite clinics to implementing a strategy to achieve a higher HU response. The total number of clinic visits a patient completed in a year was associated with a better HU response. The academic center has the ability to reschedule patients on a near daily basis; however, we conduct satellite clinics monthly to every three months depending on the satellite clinic location. Therefore, improving HU response by targeting more frequent clinic visits may remain a systematic barrier for institutions that conduct outreach sickle cell clinics at scheduled intervals. Promising approaches for satellite clinics would be to incorporate additional telemedicine videoconferences to review HU adherence or apply novel pharmacokinetic technology that can identify an initial maximal tolerated dose rather than dose titration at interval clinic visits.

Patients that admit to poor adherence present a unique challenge to dosing of $\mathrm{HU}$ at institutions that titrate to a maximal tolerated dose by targeting mild myelosuppression. Clinicians can either increase a dose of HU despite admitted poor adherence or promote adherence prior to making dose adjustments for myelotoxicity. At our institution, clinicians may defer dose adjustment aimed at mild myelosuppresion until adherence improves. However, without a systematic approach to improve adherence, our center likely maintains lower dosing and lower $\mathrm{HbF}$ response as compared to other centers. Recent advances in technology have been developed and tested in pilot studies to improve patient adherence. ${ }^{9-12}$ These strategies present novel opportunities to improve adherence in centers currently lacking a standardized approach to improved adherence.

Literature has demonstrated the association of poverty, negative socioeconomic factors and poor health outcomes in children. ${ }^{13-15}$ Our data show that our sickle cell patients cared for in our satellite clinics live in zipcodes with lower socioeconomic indicators. This highlights the need to improve adherence to HU at maximal tolerated dose in order to reduce disparities in acute care for children living with sickle cell anemia in rural or lower socioeconomic neighborhoods without immediate access to local pediatric hematology experts. Our data shows similar HU dosing and response to therapy utilizing a telehealth monitoring of laboratory values with phone call dose adjustments by a nurse clinician. Therefore, interventions to improve adherence to $\mathrm{HU}$ in Alabama can be developed and tested in satellite clinics using telehealth HU dose adjustments.

While our data demonstrated that telehealth dosing allowed a similar level of HU response among academic and satellite clinic patients, our study has a few limitations worth noting. To determine hospitalizations and ED visits, we abstracted data from patient recall in the clinic note and our institutional EMR. Our data showed a similar number of hospitalizations between academic center and satellite clinic patients but a higher number of acute care visits among academic center patients. This may represent recall bias of acute care visits among satellite clinics as we do not confirm interval history of acute care visits with local emergency care centers. However, we often receive records or transfers to our hospital for patients admitted with a sickle cell event. Second, we were unable to accurately determine from the medical records whether a patient was continued at a $\mathrm{HU}$ dose despite a higher ANC based on poor adherence. Therefore, we could only provide anecdotal evidence that some clinicians in our practice do not aggressively titrate HU in the setting of admitted poor adherence. Finally, we do not collect patient specific socioeconomic indicators as standard of care and therefore relied on zipcode to perform aggregate data analysis. 
As acceptance and $\mathrm{HU}$ usage increases at academic centers, research must continue to focus on ensuring dissemination of $\mathrm{HU}$ to patients with sickle cell living in rural areas. This data suggests that telehealth or telemedicine capacity can be implemented to monitor and titrate HU therapy in rural populations. Clinicians should not be deterred from implementing a telehealth strategy in rural areas or consider the lack of immediate access to lab results a barrier to telehealth. Future data can also evaluate outcomes of HU dosing response at academic centers implementing telehealth during the COVID-19 pandemic.

\section{Conflict of Interest Statement}

The authors have no relevant conflicts of interest related to this manuscript. Sarah Shaner MSIII received funding from a Physician Scientist Development Short Term Institutional Research Training (NIH T35 SHIRT) HLA007473.

\section{Acknowledgements}

The authors would like to thank the sickle cell clinical team for providing excellent care and the patients living with sickle cell.

\section{References}

1. Telfair J, Haque A, Etienne M, Tang S, Strasser S. Rural/urban differences in access to and utilization of services among people in Alabama with sickle cell disease. Public Health Rep . 2003;118(1):27-36.

2. Hamm J, Hilliard L, Howard T, Lebensburger J. Maintaining High Level of Care at Satellite Sickle Cell Clinics. J Health Care Poor Underserved . 2016;27(1):280-292.

3. John CC, Opoka RO, Latham TS, et al. Hydroxyurea Dose Escalation for Sickle Cell Anemia in SubSaharan Africa. N Engl J Med . 2020;382(26):2524-2533.

4. McGann PT, Niss O, Dong M, et al. Robust clinical and laboratory response to hydroxyurea using pharmacokinetically guided dosing for young children with sickle cell anemia. Am J Hematol . 2019;94(8):871879 .

5. George A, Dinu B, Estrada N, et al. Novel dose escalation to predict treatment with hydroxyurea (NDEPTH): A randomized controlled trial of a dose-prediction equation to determine maximum tolerated dose of hydroxyurea in pediatric sickle cell disease. Am J Hematol . 2020.

6. Estepp JH, Smeltzer MP, Kang G, et al. A clinically meaningful fetal hemoglobin threshold for children with sickle cell anemia during hydroxyurea therapy. Am J Hematol . 2017;92(12):1333-1339.

7. Ware RE. How I use hydroxyurea to treat young patients with sickle cell anemia. Blood . 2010;115(26):53005311.

8. Ware RE, Helms RW, Investigators SW. Stroke With Transfusions Changing to Hydroxyurea (SWiTCH). Blood . 2012;119(17):3925-3932.

9. Alberts NM, Badawy SM, Hodges J, et al. Development of the InCharge Health Mobile App to Improve Adherence to Hydroxyurea in Patients With Sickle Cell Disease: User-Centered Design Approach. JMIR Mhealth Uhealth . 2020;8(5):e14884.

10. Badawy SM, Thompson AA, Liem RI. Technology Access and Smartphone App Preferences for Medication Adherence in Adolescents and Young Adults With Sickle Cell Disease. Pediatr Blood Cancer . 2016;63(5):848-852.

11. Creary S, Chisolm D, Stanek J, et al. Measuring hydroxyurea adherence by pharmacy and laboratory data compared with video observation in children with sickle cell disease. Pediatr Blood Cancer . 2020;67(8):e28250.

12. Creary S, Chisolm DJ, O'Brien SH. ENHANCE-(Electronic Hydroxyurea Adherence): A Protocol to Increase Hydroxyurea Adherence in Patients with Sickle Cell Disease. JMIR Res Protoc . 2016;5(4):e193. 
13. Power-Hays A, Li S, Mensah A, Sobota A. Universal screening for social determinants of health in pediatric sickle cell disease: A quality-improvement initiative. Pediatr Blood Cancer . 2020;67(1):e28006.

14. Andrist E, Riley CL, Brokamp C, Taylor S, Beck AF. Neighborhood Poverty and Pediatric Intensive Care Use. Pediatrics . 2019;144(6).

15. Beck AF, Huang B, Simmons JM, et al. Role of financial and social hardships in asthma racial disparities. Pediatrics . 2014;133(3):431-439.

\section{Legend}

TABLE 1: Overall patient population

TABLE 2: Birmingham vs satellite clinic hematologic outcomes

TABLE 3: Birmingham vs satellite clinic healthcare utilization

TABLE 4: Birmingham vs satellite clinic socioeconomic outcomes

\section{Hosted file}

TABLE 1.pdf available at https://authorea.com/users/412486/articles/521179-impact-oftelehealth-visit-and-socioeconomic-status-ses-on-hydroxyurea-response-in-sickle-cellanemia

\section{Hosted file}

TABLE 2.pdf available at https://authorea.com/users/412486/articles/521179-impact-oftelehealth-visit-and-socioeconomic-status-ses-on-hydroxyurea-response-in-sickle-cellanemia

\section{Hosted file}

TABLE 3.pdf available at https://authorea.com/users/412486/articles/521179-impact-oftelehealth-visit-and-socioeconomic-status-ses-on-hydroxyurea-response-in-sickle-cellanemia

\section{Hosted file}

TABLE 4.pdf available at https://authorea.com/users/412486/articles/521179-impact-oftelehealth-visit-and-socioeconomic-status-ses-on-hydroxyurea-response-in-sickle-cellanemia 